\title{
per citare:
}

URBANI, C. (2014). La Riflessività nei contesti prescolastici. Un dispositivo di ricerca sulla professionalità insegnante. Educational Reflective Practices, I- 2014, Milano: Franco Angeli,

pp.182- 195.

\section{La riflessività nei contesti prescolastici}

\section{Un dispositivo di ricerca sulla professionalità insegnante}

\author{
Chiara Urbani \\ chiara.urbani@unive.it
}

\section{Riassunto}

I contesti prescolastici stanno suscitando sempre più interesse in relazione alla qualificazione dei sistemi educativi, richiedendo un investimento sulla qualificazione professionale degli insegnanti. Ma può un modello centrato sulle competenze professionali rispondere ai bisogni inclusivi emergenti dai contesti allargati? La proposta di qualificare il dispositivo della riflessività in funzione capacitativa sull'agency professionale dell'insegnante consente di ricondurre le opportunità formative allargate a significatività e valore personale, definendo nuovi percorsi inclusivi di formazione professionale. 


\title{
Reflexivity in pre-primary context A device for research on teacher's professionalism
}

\begin{abstract}
The pre-primary context are attracting more and more interest in relation on the qualification of educational systems, requiring an investment on the professional teachers' qualifications. May a model centred on professional skills answer at inclusive needs arising from enlarged contexts? The proposal to qualify the reflexivity device as a capability function on professional teachers' agency allows to bring educational and enlarged opportunities into significance and personal value, defining new inclusive paths for professional training.
\end{abstract}

Parole chiave: riflessività, educazione prescolastica, sviluppo professionale insegnante, formazione continua degli insegnanti, capacitazione, contesti d'apprendimento allargati.

Key- words: reflexivity, pre-primary education, professional teacher's development, in-service teacher's training, capability, enlarged learning context.

\section{Educazione prescolastica e professionalità}

Nelle politiche formative europee crescente attenzione sta assumendo il tema dell'educazione prescolastica, interpretata come il dispositivo principale per rimuovere lo svantaggio socio-educativo e prevenire l'abbandono scolastico, al fine di ottimizzare i risultati di apprendimento degli allievi e il loro inserimento occupazionale (COM 2010; COM- Eurydice, 2009; UNICEF, 2008 ).

L'investimento sulla qualificazione professionale del personale insegnante diviene la leva per migliorare i sistemi d'istruzione e formazione e le prestazioni scolastiche (COM- Eurydice, 2013; OECD- TALIS, 2009), da cui la preoccupazione di riuscire ad attrarre e trattenere personale adeguatamente qualificato, così come di sviluppare competenze professionali 
significative (COM, 2011, p. 9). Tuttavia accade che: tali politiche tendano a sottovalutare alcune componenti essenziali che intervengono a definire la qualità dei processi educativi, come quelle relazionali, professionali ed extrascolastiche, con gli stakeholders educativi della comunità sociale. La qualificazione professionale degli insegnanti andrebbe rivolta a promuovere l'esercizio di competenze di tipo strategico, non solo di tipo educativo ma soprattutto relazionale, organizzativo e gestionale (OECD- Education at a Glance, 2013) ${ }^{1}$, al fine di rispondere adeguatamente ai nuovi bisogni inclusivi emergenti, sia educativi che sociali.

In funzione dell'adeguamento e sviluppo della professionalità prescolastica, la ricerca si confronta con la definizione di nuove competenze considerate strategiche rispetto ad obiettivi di inclusività sociale.

Un importante studio internazionale su 15 paesi europei ha esaminato le competenze richieste nell'ambito dell'educazione infantile, rilevando la scarsa definizione esistente sia dei profili occupazionali che dei relativi curricoli formativi (Urban et al., 2011) ${ }^{2}$. Il modello di profilo professionale ipotizzato dai ricercatori prevede una formazione iniziale centrata sull'acquisizione di competenze minime essenziali tra loro integrate (es., tra competenze di cura e competenze educative), necessarie allo svolgimento della professione, che lasci tuttavia spazio sia ad integrazioni successive che a declinazioni specifiche ed adattamenti contestuali, secondo una prospettiva processuale ed evolutiva. Tale profilo trova il suo riferimento fondamentale nelle pratiche riflessive, capaci di ripensare l'azione professionale adattandola alla specificità contestuale, ma apre parallelamente una nuova prospettiva di autonomia decisionale, di responsabilità di scelta e di gestione della complessità (Tessaro, 2012).

L'indagine ha inoltre riscontrato un ulteriore limite dei profili professionali analizzati: essi risultano spesso incentrati sullo sviluppo delle competenze relative alla relazione tra professionista (educatore/ insegnante) e bambino/i, mentre risultano essere quasi completamente trascurate le com-

$\left({ }^{1}\right)$ Il rapporto OECD del 2013 sottolinea il fatto che gli insegnanti italiani dedichino la maggior parte del loro tempo di lavoro all'insegnamento nelle classi e poco tempo a scuola per l'organizzazione, per altre attività con gli studenti ecc... Questo aspetto dell'attività docente si traduce in un certo tipo di didattica, che secondo il Report rende i quindicenni italiani molto meno brillanti nei test internazionali rispetto, ad esempio, dei loro coetanei giapponesi. Diventerebbe pertanto indispensabile considerare dapprima un cambiamento alivello di didattica e dell'organizzazione del tempo scuola, e quindi formulare una proposta coerente din aumento del tempo-scuola dedicato ad altre attività oltre l'insegnamento in classe.

$\left(^{2}\right)$ La ricerca europea CoRe (Competence Requirements in Early Childhood Education and Care) ha intrapreso uno studio sulle competenze formali richieste dalla qualificazione formativa della professionalità prescolastica. I risultati segnalano l'efficacia di percorsi di formazione continua diversificati, oltre ad evidenziare come la qualità della professionalità non possa essere interpretata solo in senso individuale, in quanto risultante di processi di interazione sociale più allargati rispetto alla sola comunità scolastica, che definiscono il sistema formativo integrato come "sistema competente". 
petenze relative alla relazione tra operatori (lavoro d'èquipe) e con i genitori.

Emerge quindi con chiarezza il fatto che il concetto di competenza professionale non possa essere più ricondotto al possesso e alla traduzione pratica di abilità/ conoscenze di tipo specifico, focalizzate esclusivamente sulla relazione educativa, quanto piuttosto:

[...]'competence' in the early childhood education and care context has to be understood as a characteristic of the entire early childhood system. The competent system develops in reciprocal relationships between individuals, team, institutions and the wider socio-politcal context. A key feature of a 'competent system' is its support for individualsis to realise their capability to develop responsible and responsive practices that respond to the needs of children and families in everchanging societal context (Urban et al., 2011, p. 20).

L'esigenza di includere i contesti allargati (parentali, intergenerazionali, sociali) nel discorso educativo rende necessaria l'attivazione di dispositivi riflessivi, in funzione del ripensamento, elaborazione e ri-modulazione di approcci e prospettive, e della costruzione di nuova conoscenza, contribuendo alla ri-definizione della professionalità insegnante. La riflessività infatti non funge solo da principio di adattamento di pratiche ed interventi, ma assume il ruolo di dispositivo formativo essenziale, capace di sviluppare competenze di ordine superiore in relazione all'aumento di autonomia e responsabilità professionale. ${ }^{3}$

Un'altra ricerca condotta in Slovenia ha cercato di individuare la correlazione in termini formativi tra la certificazione delle competenze degli insegnanti prescolastici sugli ISSA standard ${ }^{4}$ e l'auto- valutazione professionale (Vonta, 2004). Esaminando il rapporto tra i risultati ISSA standard con alcune variabili (es. percorsi di istruzione formale, numero di anni di esperienza di insegnamento, etc.) si è giunti ad identificare come la "quantità" della formazione in servizio risulti positivamente correlata ai punteggi ISSA, a fronte di un'assenza di correlazione tra il livello di istruzione formale del docente e i punteggi ISSA. Premessa l'inadeguatezza di percorsi

$\left(^{3}\right)$ Tessaro (2012), nel suo articolo Lo sviluppo della competenza. Indicatori e processi per un modello di valutazione assegna alla competenza un valore processuale ed inseparabile tra pensiero ed azione, teoria e pratica, conoscenza ed esperienza, che si sviluppa mediante processi prattivi e retroattivi ininterrotti coi contesti d'uso e le situazioni specifiche in cui prende forma, per cui non risulta riducibile ad un processo uniforme e lineare.

$\left({ }^{4}\right)$ L'International Step by Step Association (ISSA) ha definito 5 standard di competenza collegati alla professionalità prescolastica: Individualizzazione, Learning Environment, Partecipazione della Famiglia, Strategie didattiche per l'apprendimento significativo, Pianificazione e valutazione, Sviluppo Professionale. 
di formazione iniziale di tipo marcatamente disciplinare, sorprende tuttavia che ad incidere sullo sviluppo delle competenze risulti più significativo il valore della formazione continua "in servizio" rispetto al valore di quella iniziale.

Il professionista, infatti, sviluppa competenza ad agire nella continua interazione con l'esperienza dei contesti informali di esercizio della professionalità, che stimolano l'attivazione di dispositivi riflessivi in relazione alle componenti di: indagine sulla realtà in funzione della risoluzione dei problemi (ripensamento e modulazione della sua azione educativa per meglio adattarla alla situazione), costruzione e adeguamento della conoscenza e dei processi conoscitivi per orientare la propria scelta, ri-costruzione dell'esperienza per interpretarla e meglio comprenderla alla luce dello svelamento delle premesse di pensiero (Striano, 2001, p. 130).

Quindi i percorsi di tipo informale, che l'insegnante realizza al di fuori di quelli formali e/o istituzionali intenzionalmente formativi, assumono un valore crescente in relazione alla loro capacità di costruire competenze di tipo socio-interpretativo, relazionale e comunicativo, strategico- organizzativo, attraverso la mediazione riflessiva. La sua capacità generativa di significatività dei rapporti di interdipendenza ed interazione tra fatti ed esperienze identifica il contesto della formazione continua "in servizio" come il più proficuo rispetto alla capacitazione di competenze professionali strategiche.

Dalle ricerche esaminate emerge dunque un quadro della professionalità educativa prescolastica contrassegnato da questioni incalzanti, a cui non sembra più possibile rispondere in termini di professionalità "tradizionale", derivata cioè da una cultura della standardizzazione che replica modelli formativi centrati sull' acquisizione di competenze "sicure”, volte a padroneggiare al meglio $i$ processi di insegnamento-apprendimento. L'evoluzione dei contesti socio-istituzionali e delle conoscenze esige lo sviluppo di una professionalità dinamica (capace di intraprendere processi di autodeterminazione in ordine allo sviluppo e all'attivazione delle competenze), ed innovativa (capace di proporsi quale riferimento esemplare e propulsivo di nuovi approcci).

La variegatezza ed eterogeneità dell'esperienza professionale allargata richiede la costruzione di dispositivi riflessivi capaci di problematizzare la realtà, dotarla di senso e costruire strategie d'azione di tipo inclusivo, al fine di aumentare l'autonomia di scelta e di conduzione responsabile.

I contesti dell'educazione prescolastica, in relazione alle opportunità formative e capacitative generate dalle relazioni e dai contesti professionali informali, appaiono i più significativi e qualificanti rispetto allo sviluppo di competenze strategico- riflessive, in funzione della definizione di una nuo- 
va politica di investimento sulla formazione continua in senso inclusivo della professionalità docente.

\section{Riflessività e capacitazione}

L'aspetto pratico e processuale rivestito dalla riflessività in rapporto alla qualificazione professionale è stato ben indagato da Mortari (2010) tramite una ricerca basata su interviste realizzate ad un campione di 35 insegnanti, allo scopo di mettere in luce le azioni e i processi riflessivi attivati nella quotidianità scolastica. Attraverso l'analisi delle descrizioni degli insegnanti, l'autrice individua non solo l'ambito dell'agire "in classe", ma rileva anche le cosiddette condizioni facilitanti l'agire (stare in dialogo con la situazione; essere insegnanti in ricerca; agire al plurale; creare alleanze educative con le famiglie) intese quali esperienze pratiche e riflessive fondamentali, capaci di influenzare in modo decisivo l'azione professionale.

La riflessività condizionale sull'agire professionale si esprime in una serie di comportamenti che denotano atteggiamenti mentali positivi e metacognitivi, nonchè dialogici, di "reciprocità supportiva",5. Tali "componenti condizionali" dell'agire professionale sembrerebbero derivare dall'interazione delle capacità personali con fattori intervenienti, simili a quelli che Nussabum (2012) identifica nei termini di fattori di conversione di tipo ambientale, esperienziale e situazionale, che innescano e attivano capacità interpretative e rielaborative superiori, gradi di consapevolezza e autonomia maggiori, che determinano quelle "capacità combinate" in grado di perseguire il raggiungimento dei funzionamenti.

Solo la combinazione delle capacità interne con fattori di conversione di tipo politico, sociale, culturale ed economico determina lo sviluppo di capacità combinate di tipo superiore, che conferiscono maggiore libertà sostanziale (non solo di scegliere, ma, anche, di conseguire) di realizzare effettivamente i funzionamenti prescelti.

Quindi non si tratta di garantire il possesso delle capacità interne ma anche di creare le condizioni esterne favorevoli alla loro estrinsecazione:

[...] per garantire una capacità a una certa persona non è sufficiente produrre stati interni di disponibilità ad agire. È almeno altrettanto necessario predisporre

$\left({ }^{5}\right)$ Le componenti condizionali sull'azione professionale possono essere indicate in termini di: l'immaginazione anticipatrice che aiuta a prefigurare l'azione e a costruire contesti generativi, la riflessività esercitata in ogni aspetto della vita personale (il "pensare altrove”, il "dialogo tra sè e sè”) per lasciarsi provocare dall'intuizione e dal pensiero divergente, il lasciarsi sorprendere dal contingente per accogliere l'inatteso con disponibilità, la capacità di lasciarsi “formare e trasformare” nella relazione con gli altri, ed altre ancora, sono tutte disposizioni e atteggiamenti mentali che denotano apertura e fiducia nei confronti delle sollecitazioni del reale (Mortari, 2010). 
l'ambiente materiale e istituzionale in modo che le persone siano effettivamente in grado di funzionare. Dovremmo dunque andare al di là dell'attenzione specifica alla realizzazione di capacità interne, com'è tipico dell'istruzione, per focalizzarci sulla capacitazione sociale delle opportunità di realizzare combinazioni alternative di funzionamenti. (Nussbaum, 2012, p. 108).

Le capacità combinate dell'insegnante favoriscono il funzionamento dello sviluppo professionale attraverso l'interazione con i fattori di conversione rappresentati dai contesti d'apprendimento allargati e le relazioni informali, le opportunità di network e di governance con soggetti diversi (fattori sociali), dalle garanzie e norme contrattuali e istituzionali e dai piani di carriera (fattori normativo-istituzionali), dall'esistenza di comunità di pratica professionali e contesti organizzativi (fattori organizzativi e di processo), dalle opportunità di sviluppo professionale e formazione continua, nonchè di mobilità interprofessionale e/o internazionale (fattori di risorse).

Tali fattori di conversione influenzano in modo determinante lo sviluppo delle “componenti condizionali” dell'esercizio pratico della riflessività quali quelle segnalate da Mortari (2010). La disponibilità dei fattori di conversione, unita alla mediazione operata dalla riflessività, determina l'attivazione di processi di capacitazione, cioè di libertà di scegliere tra opzioni alternative di funzionamenti (Sen, 2000). Le opportunità di carattere socio-culturale, organizzativo-istituzionale e personale diventano concrete nel momento in cui non solo si rendono disponibili, ma nel momento in cui si attivano tramite processi di riflessività espansivo- ricorsiva (Margiotta, 2010), fungendo da "amplificatori euristici" del loro valore potenziale. Ovvero, i fattori di conversione diventano efettivamente capacitativi solo nel momento in cui intervengono dispositivi riflessivi capaci di ricondurre le opportunità offerte a valore personale e decisionale.

La riflessività assume dunque il ruolo di dispositivo-cardine di capacitazione delle opportunità, che acquistano valore e significatività personale solo nella misura in cui vengono autenticamente e responsabilmente scelte in funzione dei propri obiettivi, a cui contribuiscono a seconda del livello di congruenza e responsività che sono in grado di esprimere. Ad esempio, un programma di formazione per gli insegnanti prescolastici diventa un'opportunità concreta di miglioramento professionale solo nel momento in cui viene effettivamente convalidato da pratiche riflessive di progettazione, riflessione in azione e di conversazione riflessiva con la situazione (Schön, 1993), coinvolgendo l'intera collegialità nella definizione degli obiettivi, come esercizio della libertà sostanziale di scegliere e negoziare socialmente il percorso considerato più efficace al conseguimento dei propri obiettivi di formazione professionale. 
Se dunque il valore fondante la professionalità consta nella libertà di scegliere i propri fini realizzativi ed agire in coerenza con essi, la riflessività si dimostra tanto più efficace quanto più permette di attivare processi di capacitazione decisionale e realizzativa, individuale e sociale, capaci non solo di significare entro un processo autenticamente scelto le opportunità di conversione disponibili, ma soprattutto di attivarle per produrre cambiamento e trasformazione delle prassi. Tali processi determinano l'agency professionale, intesa come un "dare forma" ai propri obiettivi d'azione (Sen, 2000). La riflessività riveste quindi una funzione formativa cruciale sulla formazione, costituendo il dispositivo privilegiato di capacitazione dell'agency professionale.

\section{Un ipotesi di ricerca: dalla riflessività all'agency professionale}

La libertà di agency consiste nella possibilità garantita al docente di scegliere ed esprimere la propria azione in funzione del perseguimento di qualsiasi obiettivo o valore che egli reputi importante. La possibilità di agire secondo libertà di agency determina inoltre la valutazione e qualificazione degli assetti sociali ed istituzionali più efficaci a favorire l'espansione delle opportunità di praticabilità (Alsop et al., 2006).

La teoria dell'agency di Sen (2000) e le ricerche svolte da Alkire (2005) ci permettono di inquadrare lo sviluppo professionale in relazione all'azione individuale, non necessariamente rivolta al raggiungimento di una finalità eticamente e socialmente determinata a livello formale- istituzionale, e nemmeno rivolta a scopi di cui si considera la giustezza ed ammissibilità etico- morale, bensì in riferimento ad obiettivi personalmente scelti entro la disponibilità di diverse opzioni alternative di azione individuale. A tal proposito, Sen (2000) puntualizza che la validità e "ammissibilità” sociale dell'azione individuale deriva dai processi di partecipazione e negoziazione democratica, che determinano i relativi processi di regolazione e legittimazione sociale.

L'anello di congiunzione tra l'agency e il funzionamento è rappresentato dalla capacitazione. La capacitazione rimanda sia all'esistenza di più opportunità/ alternative possibili, intese come opzioni di scelta differenti, in cui una persona esprime la propria libertà di scelta e di azione in relazione alle risorse possedute, sia alla capacità di attivare effettivamente quelle opportunità, in modo da conseguire gli obiettivi prescelti.

Dall'analisi dei report nazionali e internazionali sulle politiche prescolastiche e della formazione professionale emerge come la necessità di rispondere a bisogni sempre più complessi e diversificati di ordine sociale neces- 
siti dello sviluppo di competenze inclusive all'interno della professionalità docente. Tali obiettivi non risultano più conseguibili tramite una formazione centrata su competenze standardizzate, bensì richiedono maggiori livelli di autonomia e responsabilità che esigono il coinvolgimento e l'attivazione di componenti di capacitazione personale e professionale insieme.

L'ipotesi di ricerca si propone di individuare nella dimensione della libertà di agency il dispositivo funzionale di realizzazione della capacitazione professionale (sviluppo professionale). Ovvero: all'aumentare di libertà di agency aumenta anche la qualificazione dello sviluppo professionale docente. La capacitazione professionale viene intesa come la possibilità/ capacità di raggiungere al meglio gli obiettivi (funzionamenti) prescelti.

Tale possibilità si esprime nella libertà/ capacità di scegliere tra diverse opportunità/ opzioni di scelta alternative e di significarle nel proprio percorso realizzativo: la riflessività interviene nella misura in cui consente di significare tali opportunità nel proprio percorso realizzativo, per conseguirle concretamente in termini di funzionamenti.

Si tratta dunque di capire come il dispositivo della riflessività possa intervenire a migliorare l'agency professionale, che è indipendente dal possesso delle competenze e dalla loro qualità. La capacità della riflessività di coinvolgere ed attivare le opportunità di conversione e promuovere le componenti condizionali all'azione professionale determina reale cambiamento delle pratiche e influisce sullo sviluppo professionale in senso trasformativo, producendo cambiamento ed innovazione.

La pista di ricerca a seguire propone un modello di indagine centrato sulle competenze riflessive che l'insegnante attiva nell'interazione con le famiglie, da cui individuare i collegamenti con l'agency professionale. L'interazione, anche a livello informale, con la componente genitoriale permette di ripensare le modalità comunicative e di gestione della relazione che gli insegnanti mettono in atto nella loro pratica quotidiana, permettendo loro di attivare nuove opzioni di agency professionale, più proficue ed efficaci, dalle positive ricadute in senso formativo.

\section{Competenza riflessiva sull' interazione}

Essere in grado di riflettere e modificare le pratiche di interazione con i genitori/ famiglie:

\begin{tabular}{|c|c|}
\hline Conoscenze teoriche: & Livelli (Tessaro, 2012): \\
\hline 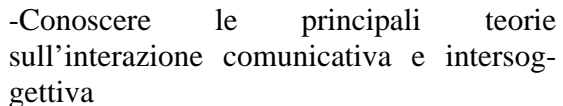 & $\begin{array}{l}\text { I.Esordiente (imitazione consapevole): } \\
\text { Applicare modalità riflessive e comuni- } \\
\text { cativo-informative basate su indicatori pre- }\end{array}$ \\
\hline
\end{tabular}


-Conoscere le teorie sulla riflessività in relazione all'esperienza (Dewey, Schön, Habermas, Mezirow)

\section{Conoscenze pratiche:}

-Saper ascoltare, interpretare messaggi e riconoscere situazioni

-Saper riflettere sulle esperienze e le relazioni di causa-effetto

-Saper comprendere bisogni ed esigenze specifiche

-Saper comunicare efficacemente

Abilità cognitive:

-Esprimere e manifestare disponibilità, apertura ( sospensione di giudizio aprioristico)

-Mantenere atteggiamenti di rispetto, attenzione, considerazione

-Mantenere autorevolezza professionale

-Adottare modalità empatiche

Abilità operative:

Riflettere e mettere in discussione pratiche, approcci, strategie comunicative e relazionali

-Elaborare strategie di riflessione collegiali condivise efficaci

-Scegliere e condividere una strategia educativa e comunicativa comune (coerenza del messagio a livello collegiale)

-Valutare la qualità della relazione e il suo andamento cedentemente codificati

II. Praticante (adattamento al contesto):

Sviluppare e mantenere rapporti positivi di collaborazione ed interazione comunicativa basati sull'analisi della situazione, sul supporto e comprensione, sul rispetto delle funzioni specifiche

III. Standard (realizzazione diretta allo scopo):

Sviluppare pratiche di riflessione cooperativa sui processi di interazione con le famiglie attraverso l'osservazione, la discussione e l'azione collegiale, regolando modalità e strategie relazionali

IV. Rilevante (specificità personale):

Sviluppare pratiche riflessive individualizzate sull'interazione famigliare (riflessione sull'esperienza) e pratiche di significazione delle esperienze a livello collegiale per meglio progettare e realizzare strategi personalizzate condivise

V. Eccellente (innovazione creativa)

Riflettere sulla propria azione in situazione in modo critico e collegiale, rivedendo le premesse iniziali qualora risultino inadeguate per adattare e/o produrre strategie d'interazione di tipo sperimentale e innovativo.

\begin{tabular}{|l|l|l|}
\hline \multicolumn{1}{|c|}{ Indicatori di Agency } & \multicolumn{1}{|c|}{ Capacitazione } & \multicolumn{1}{|c|}{$\begin{array}{l}\text { Funzionamenti profes- } \\
\text { sionali: }\end{array}$} \\
\hline -Accesso alle informazioni & $\begin{array}{l}\text {-Formulare valutazioni } \\
\text { attendibili e realistiche } \\
\text { sulla situazione }\end{array}$ & $\begin{array}{l}\text {-Stabilire rapporti di aiuto } \\
\text { e sostegno genitoriale }\end{array}$ \\
\hline $\begin{array}{l}\text {-Controllo sui processi comu- } \\
\text { nicativi, relazionali, fiduciari }\end{array}$ & $\begin{array}{l}\text {-Essere capaci di condivi- } \\
\text { dere e coinvolgere le fa- } \\
\text { miglie nella discussione } \\
\text { sull'educazione } \\
\text {-Sostenere le famiglie nei } \\
\text { processi di partecipazione, } \\
\text { inclusione, collaborazione }\end{array}$ & $\begin{array}{l}\text {-Aumentare opportunità } \\
\text { di relazione e condivisione } \\
\text { tra famiglie }\end{array}$ \\
\hline
\end{tabular}




\begin{tabular}{|c|c|c|}
\hline $\begin{array}{l}\text {-Controllo sui processi di dia- } \\
\text { logo e confronto }\end{array}$ & $\begin{array}{l}\text {-Potenziare la funzione } \\
\text { educativa familiare e/o } \\
\text { parentale } \\
\text {-Considerare e discutere } \\
\text { punti di vista differenti } \\
\text { sull'educazione } \\
\text {-Offrire e condividere il } \\
\text { proprio sapere professio- } \\
\text { nale }\end{array}$ & $\begin{array}{l}\text {-Formulare problemi ed } \\
\text { elaborare soluzioni collet- } \\
\text { tivamente } \\
\text {-Valorizzare la funzione } \\
\text { educativa professionale } \\
\text { (esperto dei processi for- } \\
\text { mativi) }\end{array}$ \\
\hline $\begin{array}{l}\text {-Promuovere autonomia deci- } \\
\text { sionale } \\
\text {-Dimostrare consapevolezza ed } \\
\text { autorevolezza } \\
\text {-Autonomia di dominio speci- } \\
\text { fico della funzione educativa } \\
\text { (esperto dei processi formativi) } \\
\text {-Favorire l'elaborazione di } \\
\text { nuove modalità educative e } \\
\text { organizzative genitoriali e fa- } \\
\text { miliari }\end{array}$ & $\begin{array}{l}\text {-Promuovere nuove vision } \\
\text { e approcci innovativi alle } \\
\text { famiglie }\end{array}$ & $\begin{array}{l}\text {-Determinare cambiamen- } \\
\text { ti sulla vita delle famiglie e } \\
\text { del bambino }\end{array}$ \\
\hline
\end{tabular}

Il disegno di ricerca prevede di individuare le competenze (riflessive) autopercepite che gli insegnanti reputano essenziali nell'azione professionale, oltre a quelle che giudicano migliorabili in funzione dei bisogni di inclusività emergenti, e/o sviluppate in percorsi formativi. La rilevazione tramite questionari permette di identificare le aree di criticità e/o coincidenza tra competenze e opportunità/ dimensioni di conversione (sociali, normativo- istituzionali, organizzativo- processuali, delle risorse) che forniscono una prima ricognizione rispetto agli ambiti di capacitazione professionale.

Successivamente si prevede l'attivazione di un contesto di ricercaazione per osservare la correlazione tra le competenze e agency (prendendo quale riferimento gli indicatori di agency), utilizzando il dispositivo della riflessività applicata alle pratiche dell'azione professionale. 
Infine, andrebbe realizzato un momento di ripensamento e significazione dell'esperienza di ricerca-azione attraverso dei colloqui individuali di tipo narrativo con gli insegnanti coinvolti per indagare la capacitazione attivata, e per formulare ipotesi di significazione individuale e professionale delle dimensioni di conversione.

In tal modo ci si propone di:

- Individuare le dimensioni di capacitazione professionale docente

- Definire un modello di sviluppo e formazione professionale

- Qualificare nuove politiche di formazione professionale

\section{Ricadute sulle pratiche}

La nuova prospettiva della capacitazione professionale permette di ipotizzare la costruzione di nuove strategie di investimento sullo sviluppo professionale che vadano al di là del tradizionale valore assegnato alle competenze standard per comprendere componenti essenziali quali l'agency professionale e la riflessività, che esprimono la libertà/capacità di conseguire realmente i funzionamenti professionali e personali ritenuti importanti.

- In termini di qualificazione professionale: riuscire a estrarre un potenziale formativo dal paradigma riflessivo consentirebbe di aprire nuove prospettive di formazione continua per gli insegnanti, di tipo collettivo, dando vita a nuove forme di comunità di pratica e a nuove reti di sviluppo professionale, in grado di rafforzare l'identità collettiva della classe docente e la sua voce negoziale all'interno del dibattito politico.

Un recente lavoro di ricerca condotto sull'incremento del dialogo professionale tra gli insegnanti prescolastici australiani e i loro supervisori (in relazione alla riflessione sulle pratiche ed in funzione della definizione del curricolo formativo) ha prodotto un primo risultato in termini di benefici sulla professionalità, favorendo lo sviluppo di competenze di ricerca professionale (Simoncini et al., 2014, p. 34-35). Questo dimostra come l'attivazione di dispositivi riflessivi entro comunità di pratica professionali (Wenger, 1999) in percorsi di introduzione alla professione generi ricadute significative in termini di qualificazione professionale, generando nuove opportunità di capacitazione sull'agency docente.

- In termini istituzionali: i cambiamenti organizzativi scolastici costituiscono una nuova leva dello sviluppo professionale docente: i dirigenti scolastici potrebbero individuare (internamente al proprio Istituto, come in rete, o nel più ampio territorio di appartenenza) nuove opportunità di gestione organizzativa dei progetti scolastici promuovendo percorsi di riflessività 
sull'esperienza, capaci di generare ricadute concrete sull'azione professionale e sulla formazione continua.

La ricerca australiana sopra citata segnala un secondo impatto in termini di benefici sulla professionalità docente: la riflessione e progettazione effettuata dagli insegnanti rispetto ai quesiti da porre nel colloquio coi supervisori ha prodotto un incremento nella comprensione dei problemi e delle opportunità di risoluzione ad essi collegate, scaturenti dalle pratiche professionali. I supervisori hanno descritto le loro pratiche e le corrispondenti prospettive, discutendo in particolare l'incremento degli investimenti realizzati dall'intera comunità scolastica in termini di iniziative curricolari. Questo dimostra come gli insegnanti “esperti” considerino il supporto organizzativo come fattore essenziale di efficacia e qualificazione dell'offerta formativa, generativa a sua volta di ricadute significative sullo sviluppo professionale (Simoncini et al., 2014, p. 35).

- In termini di sistema formativo integrato: la costruzione di un sistema formativo integrato assume un valore che prescinde dai benefici sull'educazione infantile per influire sull'incremento di capacitazione sociale: la condivisione di pratiche riflessive interprofessionali e intrascolastiche con l'esterno favorisce lo sviluppo di una comunità consapevole ed educante, capace di ripensare i sistemi di valori e costruire nuovi modelli educativi. Un' indagine condotta sulla correlazione tra programmi di formazione in servizio centrati sulla comunicazione con i genitori e lo sviluppo professionale (Symeou et al., 2012) ha evidenziato le ricadute positive generate dall'utilizzo di pratiche riflessive. Applicate alla comunicazione, queste hanno provocato una trasformazione della percezione che gli insegnanti assegnano alla relazione con le famiglie, favorendo una valutazione positiva degli insegnanti rispetto allo sviluppo di competenze strategiche di tipo inclusivo, e procurando impatti significativi in termini capacitativi sulla formazione professionale.

- In termini di formazione continua: il paradigma della capacitazione dello sviluppo professionale docente favorisce il passaggio da modelli di formazione basati su interventi temporanei, spesso episodici e frammentati, dagli impatti relativi, alla progettazione di percorsi che sappiano coniugare esperienza e riflessività al fine di produrre una reale modificazione delle pratiche.

Una ricerca longitudinale condotta nel contesto statunitense ha evidenziato come l'esperienza della riflessività applicata alla pratica quotidiana degli insegnanti prescolastici nella fase di introduzione alla professionae abbia provocato prima l'emergere di credenze, quindi la loro interazione con le pratiche e infine la loro ri-definizione in termini di adeguamento dell'azione professionale (Caudle, Moran, 2012). 
L'incremento di competenza riflessiva aumenta il potenziale trasformativo in termini di empowering professionale (Alsop et al., 2006), e provoca ricadute positive in termini di ripensamento e ri-definizione dei percorsi di formazione docente, rivolti alla capacitazione dell'agency professionale.

\section{Conclusioni}

L'interazione dialogica con i contesti d'apprendimento allargati determina la costruzione di nuovi saperi e conoscenze, pratiche metodologiche ed operative di azione professionale, e nuove procedure euristiche e riflessive che si strutturano nel problematico rapporto con complesse situazioni esperienziali, esplicitando il gradiente di generatività (Costa, 2011) tra azione professionale e potenzialità personale in termini capacitativi. La nuova centralità assegnata alla riflessività come dispositivo di capacitazione permetterebbe la formulazione di un nuovo profilo professionale dell'insegnante dell'educazione prescolastica, fondato sulle competenze strategiche di relazione con i contesti allargati, ed esportabile poi anche sui livelli scolastici successivi.

Tali configurazioni esigono la progettazione di percorsi formativi che consentano di esprimere ed affrontare la complessità del reale, non più ascrivibile a forme di razionalità tecnico-strumentale declinate su modelli di competenze di spendibilità professionale. La riflessività permette di coinvolgere le competenze entro un processo di sviluppo critico-emancipativo (Habermas, 1986) che scaturisce dalla razionalità socio- comunicativa che permette di svelare incongruenze, distorsioni e contraddizioni sottostanti ai processi di conoscenza, fino ad arrivare allo svelamento dei presupposti di pensiero e premesse di significato che condizionano i processi mentali, per revisionarli in senso trasformativo (Mezirow, 2003).

In tal senso, la creazione di un sistema formativo integrato di tipo sociale allargato, centrato sull'interazione comunicativa mediata dalla riflessività permette non solo di costruire azioni professionali coerenti ed efficaci, ma di acquisire consapevolezza dei presupposti cognitivi che intervengono nella produzione degli atteggiamenti mentali, allo scopo di aumentare livelli di autonomia e responsabilità decisionale e professionale. Ciò richiederebbe lo sviluppo di politiche di formazione continua per gli insegnanti del segmento prescolastico che riconoscano e legittimino la riflessività quale principio di capacitazione professionale: la possibilità di utilizzare dispositivi riflessivi di svelamento e ripensamento delle stutture conoscitive, stili cognitivi ed operativi, schemi mentali sottostanti alle pratiche educative consente di 
attivare processi di apprendimento critico-emancipativi in grado di qualificare i professionisti dell'educazione quali reali agenti di cambiamento e trasformazione (Striano, 2001).

\section{Bibliografia}

Alkire, S. (2005). Subjective quantitative studies of human agency. Social Indicators Research, 74, pp. 217-260

Alsop, R., Bertelsen, M.F., \& Holland, J., (2006). Empowerment in Practice. From Analysis to Implementation. Washington, DC: The International Bank for Reconstruction and Development/ THE WORLD BANK.

Caudle, L., \& Moran, M.J. (2012). Changes in Understandings of ThreeTeachers' Beliefs and Practice Across Time: Moving From Teacher Preparation to In-Service Teaching. Journal of Early Childhood Teacher Education, 33(1), pp. 38-53.

COMMISSIONE EUROPEA, (2010). Comunicazione della Commissione Europa 2020, Una strategia per una crescita intelligente, sostenibile e inclusiva, COM (2010a) 2020 definitivo, Bruxelles, 03.03.2010.

COMMISSIONE EUROPEA/ EACEA/ Eurydice, (2013). Cifre chiave sugli insegnanti e i capi di istituto in Europa. Edizione 2013. Rapporto Eurydice. Lussemburgo: Ufficio delle pubblicazioni dell'Unione europea

COMMISSIONE EUROPEA, (2009). Rapporto Eurydice, Educazione e cura della prima infanzia in Europa: ridurre le disuguaglianze sociali e culturali, Bruxelles: Agenzia esecutiva per l'istruzione, gli audiovisivi e la cultura.

COMUNICAZIONE DELLA COMMISSIONE, (2011). Educazione e cura della prima infanzia: consentire a tutti i bambini di affacciarsi al mondo di domani nelle condizioni migliori. Bruxelles, 17.2.2011, $\operatorname{COM}(2011) 66$ definitivo.

Costa, M., (2011). Criticità e opportunità di sviluppo professionale del docente nei primi anni di carriera in Italia. Formazione \& Insegnamento, IX (3), pp. 42-58.

Habermas, J. (1981; 1986). Teoria dell'agire comunicativo. Bologna: Il Mulino.

Margiotta, U. (2010). Abilitare la professione docente. Lecce: Pensa MultiMedia.

Mezirow, J. (1991; 2003). Apprendimento e trasformazione. Il significato dell'esperienza e il valore della riflessione nell'apprendimento degli adulti. Milano: Cortina. 
Mortari, L. (a cura di) (2010). Dire la pratica. La cultura del fare scuola. Milano: Bruno Mondadori.

Nussbaum, M. (2012; 2011). Creare capacità. Liberarsi dalla dittatura del Pil. Bologna: Il Mulino.

OECD, (2013). Education at a Glance 2013: OECD Indicators. OECD Publishing.

OECD-TALIS, (2009). Creating Effective Teaching and Learning Environments: First Results from TALIS. Paris: OECD Publishing.

Schön, D. (1983; 1993). Il professionista riflessivo. Per una nuova epistemologia della pratica professionale. Bari: Dedalo.

Sen, A. (1999; 2000). Lo sviluppo è libertà. Perchè non c'è crescita senza democrazia. Milano: Mondadori.

Simoncini, K. M., Lasen, M., \& Rocco, S. (2014). Professional Dialogue, Reflective Practice and Teacher Research: Engaging Early Childhood Pre-Service Teachers in Collegial Dialogue about Curriculum Innovation. Australian Journal of Teacher Education, 39(1), pp. 26-44.

Symeou, L., Roussounidou, E., \& Michaelides, M., (2012). "I Feel Much More Confident Now to Talk With Parents”: An Evaluation of InService Training on Teacher-Parent Communication. School Community Journal, 22 (1), pp. 65-88.

Striano, M. (2001). La razionalità riflessiva dell'agire educativo. Napoli: Liguori.

Tessaro, F. (2012). Lo sviluppo della competenza. Indicatori e processi per un modello di valutazione. Formazione \& Insegnamento, X (1), pp. 105-119.

UNICEF Report Card n. 8, (2008). Come cambia la cura dell'infanzia. Un quadro comparativo dei servizi educativi e della cura per la prima infanzia nei paesi economicamente avanzati. Firenze: Centro di Ricerca Innocenti.

Urban, M., Vandenbroeck, M., Peeters, J., Lazzari, A., Van Laere, K. (2011). CoRe Competence requirements in Early Childhood Education and Care. Report for European Commission, DG Education and Culture.

Vonta, T. (2004). Teacher Evaluation Using ISSA Standards: A Tool for Professional Development and Quality Improvement. Educating Children for Democracy, 7 (summer/fall), pp. 21-25.

Wenger, E. (1999). Communities of Practice: Learning, Meaning, and Identity. Cambridge: Cambridge University Press. 